July 2000

\title{
Physicalism and the Fallacy of Composition
}

Crawford Elder

University of Connecticut Department of Philosophy, crawford.elder@uconn.edu

Follow this and additional works at: https://opencommons.uconn.edu/philo_articles

\section{Recommended Citation}

Elder, Crawford, "Physicalism and the Fallacy of Composition" (2000). Philosophy Articles. 3.

https://opencommons.uconn.edu/philo_articles/3 
Crawford L. Elder

Department of Philosophy, U-54 [Published in Philosophical Quarterly, 50 (2000), pp. 332-43.]

University of Connecticut

Storrs, Connecticut 06269-2054

U.S.A.

\section{Physicalism and the Fallacy of Composition}

A mutation alters the hemoglobin in some members of a species of antelope, and as a result the members fare better at high altitudes than their conspecifics do; so high-altitude foraging areas become open to them that are closed to their conspecifics; they thrive, reproduce at a greater rate, and the gene for altered hemoglobin spreads further through the gene pool of the species. That sounds like a classic example (owed to Karen Neander, 1995) of a causal chain traced by evolutionary biology. But a view now nearly universal among philosophers maintains that such biological causation is always shadowed, perhaps even rivaled, by causation on a different level. ${ }^{\text {That }}$ The subgroup of antelopes forages in areas closed to the conspecifics is a state of affairs embodied or realized, notes this view, in certain movements and state changes done by certain physical microparticles-untold billions of microparticles and movements, but a finite and determinate (more on this below) collection nevertheless. That the subgroup reproduces at a greater rate is likewise realized by a huge collection of microparticle movements, a different collection. And the microparticle happenings comprised in the first collection are causally responsible, strictly in accordance with the laws of microphysics, for the microparticle happenings in the second. Biological causation is always shadowed, perhaps even rivaled, by causation on the level of microphysics.

The view I mean is general: any case of causing uncovered by any of the special sciences can be recaptured at the level of microphysics. This view is I think what most philosophers mean by "physicalism"; in any case, "physicalism" is the label I shall use. Physicalism comes in two forms. Modest physicalism holds that any causal transaction reported by the special sciences can be retraced by

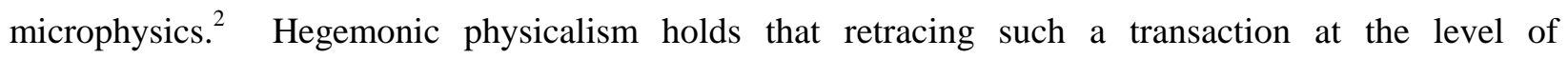


microphysics is actually reporting the only causation that is going on: the happenings identified by the special sciences only appear to cause something, and the real causing is done by large collections of microparticles. ${ }^{3}$ This paper focuses on the weaker thesis of modest physicalism. But I will bear in mind that it is perhaps less stable, ${ }^{4}$ and certainly less exciting, than hegemonic physicalism.

This paper argues that modest physicalism lacks supporting argument; a fortiori, hegemonic physicalism lacks it as well. There is no reason to suppose that causings reported by the special sciences are in general shadowed by causal transactions between one collection of microphysical events and another. This paper attributes the impression that physicalism is undeniable-modest physicalism, at least - to the fallacy of composition. It is undeniable, let us allow, that each microphysical happening comprised in a "cause" collection causes some further microphysical happening, and we can temporarily grant (though see below) that each such microphysical happening causes, by virtue of a causal chain which it launches, some individual microphysical happening comprised in the corresponding "effect" collection. It does not follow that the cause collection causes all the happenings in the corresponding effect collection, or causes a large subset of them, or causes anything. Separate argument is needed to establish those claims. None is available, I shall suggest.

This paper trades on three assumptions about causation which there is not room here to defendbut they are fairly common assumptions, and physicalists especially should be friendly towards them. The first is that the relata of causation are finely-individuated entities. I have argued elsewhere that they are states of affairs (Elder forthcoming). For the purposes of this paper, it would be just as good if they were finely-individuated events, of the sort which Jaegwon $\operatorname{Kim}(1969,1980)$ discusses or the (different) sort Stephen Yablo (1992b) defends. What they cannot be, for the purposes of this paper, are coarsely individuated events such as Davidson $(1967,1969)$ affirms. The second assumption is that every individual causing instantiates some general causal law. The third is that for a state of affairs (or Kimevent) $c$ to be a cause of effect $e$ is for it to be what Bennett (following Mackie) calls "an NS condition" of $e$ (Bennett 1988, Ch. III). That is, $c$ must be, in a way set by the causal laws of nature, a Necessary (i.e. 
indispensable) component of developments Sufficient, given the surrounding circumstances, for occurrence of $e$.

Physicalists often elect the hegemonic variant of their position, and to this extent they must be favorably disposed towards the first two of these assumptions. Without the first assumption, the collection of microparticle happenings which realizes the wider foraging by antelopes in the subgroup, and that wider foraging itself, are really one and the same event, just differently described. So whatever the former causes, the latter causes. Distinguishing these events (or states of affairs) is what opens the door to the claim of hegemony. What substantiates that claim, for many physicalists, is the further thought that the laws which underwrite causation at the level of the microparticles are far better, as laws, than the laws (putative laws, perhaps) which underwrite causation by the happenings identified by the special sciences. The laws of the special sciences, hegemonic physicalists argue, are riddled with exceptions unless protected by ceteris paribus clauses. Yet the ceteris paribus clauses seem in principle to resist precise specification, unless as "except where this laws fails to hold". This suggests, however, that the individual causings reported by the special sciences are less than genuine only if causings need to be underwritten by causal laws. So physicalists tend, and should tend, to be well disposed towards my second assumption as well.

\section{I}

But my conclusion may seem too sweeping, and not just to physicalists. Does it really never happen that an instance of causation at the level of medium-sized objects is underlain by an instance at the level of microphysics? In one particular sort of case, I admit, this does indeed happen. The burden of this paper is just to establish that it does not always happen—not in all individual causings reported by what physicalists call "the special sciences".

The sort of case in which it happens is the case in which there is a rationale, from the standpoint of microphysics, for collecting together all the microparticles which figure in realizing the event identified 
as "cause" at the level of medium-sized objects. There rarely is such a rationale, I shall argue, with the individual "cause" events reported by biology or economics (or, for that matter, sociology or behavioral psychology, etc.)

To say what such a rationale would look like, and why it matters, will require sections II and III of this paper. But a picture here may be worth many words later. I nudge an irregularly shaped rock towards the edge of a table. By pushing its center of gravity beyond the edge, I cause the rock to fall. So, at least, mechanics—and what some call "folk physics"-reports. Can this instance of causation be recaptured at the level of microphysics? That the rock's center of gravity is at $t_{l}$ above nothing but air is a state of affairs realized in the positions and motions of a myriad of microparticles. The rock's fall at $t_{2}$ is embodied in a different array of positions and motions, involving very largely the same myriad of microparticles. But plainly it does make sense, from the standpoint of microphysics, to take all the microparticles that figure in realizing the "cause" event together. For the fate of each is intertwined with the position and state of all the rest. Wherever the avalanche of microparticle events begins, that terminates in the "effect" event-in the careers of whichever microparticles—that avalanche ends up embroiling all other microparticles that figure in the "cause" event.

But with typical instances of causation reported by biology, say, or by economics, this synergythis acting in concert-disappears at the level of the microparticles. Or so I shall argue. Consider, for example, the high-grazing antelopes. Is the microphysical fate of each microparticle that figures in realizing the biologist's "cause" event intertwined with that of every other? A preliminary question is just which microparticles the question asks about—just which microparticles it is, whose movements and state changes jointly embody the state of affairs that these antelopes now forage in places not open to their conspecifics. Too many, I eventually shall argue, for it to be plausible that a collection so scattered can cause anything at all. But the start of the answer need not be controversial: namely, at the least, all those microparticles contained in the bodily boundaries of the high-grazing antelopes and their conspecifics. For otherwise the physicalist would run a risk of not circumscribing enough microparticle happenings to capture the fact that it is antelopes which differ in how they are faring. But there will then be familiar 
problems about just which microparticles to count as falling within these boundaries, and which without (Unger 1980; cf. van Inwagen 1990, p. 214 ff). How, in terms specific enough to generate a list of microparticles, is it determined where a hair in the antelope's coat leaves off and a drop of water that has begun to permeate that hair takes up? Perhaps the question can be finessed by generosity. Let the physicalist include all molecules that lie within a one-inch zone outside what common sense regards as the antelope's outer surface; let him also include all microparticles in molecules of gases in each antelope's lungs; etc. But next note that the physicalist must circle enough microparticle occurrences to embody the fact that the genetically well-favored subgroup is foraging unusually well. Successful foraging involves the ingestion of nutrients. So the microparticles in all the nutrients, contained in all the high-meadow grasses that get ingested, must be included. Then too, what about the jaw movements by which these antelopes tear and chew these nutritious grasses? If the physicalist is to capture the fact that these are not freakishly lucky events, but applications of the antelopes' normal behavioral repertoire in what is merely a new setting, perhaps enough of the evolutionary history has to be included to capture the fact that the antelopes have foraging in their behavioral repertoire - that neural routines for foraging were at one time selected for (Millikan 1993). Now the cause collection of microparticle movements starts to stretch back in time to ancestor antelopes, and to feature microparticles which no longer are in the bodies of antelopes at all. How likely is it that there will be a rationale, from the standpoint of microphysics, for collecting together the states of all these scattered microparticles as a "cause" event?

If the typical causal transactions reported by evolutionary biology look messy and sprawling, when viewed at the level of the microparticles, so too, famously, do the causal transactions reported by economics. Consider any case in which economics depicts, as the cause of some individual outcome, the fact that money is distributed in a particular economy in a particular way. Money is always physically embodied. But, as Jerry Fodor points out in a different context (Fodor 1975, p. 15), there seems to be virtually no limit to the sort of physical stuff of which money can be composed. Its status as money depends not on its physical composition but on something relational and social—on its being sanctioned to play certain roles by a society or a government. So again there is a hard question about how even to 
begin assessing the physicalist's claim-how to determine just which microparticle events fall within the shadow of the monetary distribution which economics depicts as a cause. The physicalist must assemble enough microparticle movements to recapture, at the level of the microparticles, the fact that money in this economy is distributed in such-and-such a way. For this it would evidently not be enough to count just movements of all microparticles in all actual pieces of money. One needs enough microparticle events to capture the fact that those pieces have an assigned social role. So one has to include movements and state changes in at least some microparticles in some persons, perhaps reaching well back in history. Perhaps also one must include microparticle events which jointly realize the existence of certain legal documents, certain courts of law, certain offices. How likely is it that there will be a rationale from the standpoint of microphysics for collecting all these happenings together as a cause?

To sum up: true enough, any individual occurrence warrantedly depicted by a special science as causing something has a physical embodiment. But the collection of microparticle events which jointly embodies it will typically be sprawling and disjointed, to a striking degree. That point will form the nerve of my argument against physicalism. And already we have one result: it is not really plausible to say that every last microparticle event comprised in the physicalist's "cause collection" is causally involved in bringing about the collection of microparticle events which shadows the "effect" explained by the special science. Some of them are just along for the ride, it seems. (More on this in section III.) Still, this result need not damage the physicalist's central claim. We must still ask: if the physicalist's "cause collection" is often bigger than it needs to be, to cause what it is supposed to cause, is it always at least big enough? Is it causally sufficient to bring about all the microparticle happenings which jointly realize the outcome which the special science claims causally to have explained? If so, the heart of the physicalist's position is intact. 
Should we think of the physicalist's "cause collection" as producing the corresponding "effect collection" all at once, or component microparticle event by component microparticle event? In the present section I shall consider the former suggestion, and in the next section the latter.

Imagine then that the microparticle events which embody the lucky antelopes' foraging together cause, in a single (if protracted) instance of causation, the collection of microparticle events which embodies the fact that the lucky hemoglobin gene spreads thorough the species. But how can this be, if every individual causing must instance some general law of nature? Could there really be a law of nature to the effect that whenever such-and-such movements and state changes befall microparticles of suchand-such a description — the list of these being billions of places in length—other movements and state changes, again in the billions, will befall billions of other microparticles?

The problem is not that such a law would be "general" in name only. It would be general in name only, of course. Any law which subsumed the antelopes' high-altitude foraging under a description as fine-grained as this would assuredly come into play only once in the history of the world. (It would be "exceptionless" in only an empty sense.) Nor is the problem that, because antecedent and consequent in such a law would get satisfied only once in history, we could never have warrant for believing it to be a law. For plainly the idea would be that we were warranted in a different way in believing this. The idea would be that the massively specific law is a theorem jointly entailed by simple and familiar laws of microparticle causation — laws which, for example, link a movement or state change in one microparticle with movements and state changes in a few other microparticles. Take several such simple laws together, the thinking would run, and plug in a massively complex description of antecedent conditions (capturing all or most of the "cause collection"), and out comes a massively complex description of an effect.

But why think that in addition to the simple and truly general laws of particle physics which jointly generate that theorem, that theorem is a law of nature in its own right? Such a position seems to run counter to actual scientific practice. Suppose that a given science affirms a number of separate causal 
laws governing phenomena within its domain. The separateness of the laws then depicts the transactions covered by the laws as causally independent of one another. Will that science also take there to be a law governing what happens when the antecedent in one of these separate laws takes one value, and the antecedent in another takes a second value, and the antecedent in a third takes a third value? Sometimes, but not always, and not automatically. If it turns out that specific values of the independent antecedents sum together in distinctive and characteristic ways - as when gravity and electromagnetism combine to shape the trajectory of an ion on the surface of the sun - then the science will judge there to be a separate law governing conjunctions of values for antecedents in each of the original laws. Or if it turns out that values of what looked like quite different antecedents are really variants on a single underlying phenomenon - as where the determinants of evaporation and the antecedents in Boyle's Law turned out both to be variants on molecular motion - the science will posit a new law or laws, and will take the original separate laws to have been theoretically supplanted. Either way it is crucial that the new law predict and explain phenomena not already predicted by the conjunction of the original separate laws alone. Otherwise no actual science will find warrant for positing a separate law governing conjunctions of values of antecedents in previously posited laws.

We have taken the physicalist to hold that the microparticle events composing the effect collection get produced by causal chains largely independent of one another, starting from causally independent microparticle events in the cause collection. (More on this assumption in the next section.) Each of the latter microparticle events shoots its causal influence down to one of the microparticle events in the effect collection, and the shots do not (in general) cross. No large-scale summing of causal sources occurs. No massively complex law of microphysics is needed to predict all the events in the effect collection. If such a law were formulated, it would predict nothing not already predicted by the laws which jointly ground it.

But might the physicalist rejoin that while there would be no warrant for affirming such a massively complex law, such a law might nevertheless obtain? Why suppose, the physicalist might ask, that the metaphysical question of which laws obtain in nature has close connection to the epistemological 
question of which laws we are justified in affirming? But this rejoinder would be a dangerous one for the physicalist to offer, given the assumptions from which this paper departs. It would be especially dangerous for the hegemonic physicalist. For the hegemonic physicalist takes for granted that the more closely a putative law answers to our epistemic practices - the more it is simple, sweeping, and free from apparently gerrymandered ceteris paribus clauses - the greater its claim to limn one of the ways in which events in the world really get caused. But even the modest physicalist courts danger by driving too firm a wedge between what laws may obtain and what laws are warrantedly assertible, at least provided one assumes (as this paper does) that every genuine causing must instance a law of nature. For should the physicalist concede that we could have no warrant for affirming a law linking the cause collection as a whole to the effect collection as a whole, he would thereby admit—given our assumption—-that we could have no warrant for supposing that any such causation occurs.

I conclude that physicalism is indeed unsupported-provided we take it as asserting a one-shot causation, by "the cause collection", of "the effect collection" as a whole.

III

But the physicalist can, I have just allowed, maintain that individual microparticle movements comprised in "the cause collection" bring about, via separate causal chains, each of the different microphysical happenings that compose "the effect collection". One might ask: isn't this enough? If the physicalist depicts the causing done by "the cause collection" as a piecemeal affair, involving many separate instances of causation, could she not rightly claim that in effect the cause collection produces the whole effect collection after all?

The problem with this suggestion lies not with the laws that would underwrite the causation. The laws invoked would be simple, standard laws of microphysics. For separate microparticle events within the cause collection launch, in accordance with such laws, separate causal chains, each terminating in a 
separate microparticle event within the effect collection. Between them, I have allowed, the microparticle events in the cause collection are responsible for every event within the effect collection.

No, the problem would lie in claiming, on this basis, that the cause collection itself-as a whole-causes anything. It does not help to say that the cause collection exercises piecemeal causation, causing by one chain Microparticle Event \#1 within the effect collection, by another chain Microparticle Event \#2, by yet a third chain \#3, etc. The problem is that saying that the cause collection as a whole causes Event \#1, just on the strength of a premise that this or that component within it causes Event \#1, commits the fallacy of composition. And not only is the claim logically unwarranted; it is implausible. The cause collection in the case of the antelopes, for example, comprises movements and state changes done by microparticles scattered through high-meadow grasses and low-meadow grasses, through the bodies of high-grazing antelopes and low-grazing ones, even perhaps through the bodies of ancestor antelopes long since departed. Why suppose that a collection of states of affairs involving all these farflung microparticles as a whole produces any outcome? Why isn't such a collection too disjointed to act at all?

The challenge is particularly acute if one assumes, as this paper does, that a cause of outcome $e$ is an NS condition of $e$ - a state of affairs (or event) which is, in a way fixed by the causal laws of nature, an indispensable component of circumstances which jointly suffice for $e$ 's occurrence. For consider any event within the effect collection in our example — any individual microparticle happening $e$, among those which jointly embody the eventual prevalence of antelopes with altered hemoglobin. To make matters concrete, let $e$ be a microparticle movement in the eyelid of a newborn offspring of a prolific highforaging antelope. We assume with the physicalist that some microparticle happening(s) in the cause collection launched a many-staged causal chain which resulted in $e$. But could it possibly be claimed not just that some microparticle movements within the cause collection were involved indispensably in launching this one chain, but that every last one of them was? Only so can the cause collection as a whole qualify as an NS condition for $e^{5}$ 
To see just how far-fetched a claim this would be, consider a scenario on which it would after all be true. A causal chain begins just as one of the first high-grazing antelopes bites down on a surprisingly succulent blade of high-meadow grass. What launches the chain is a movement of a microparticle within the antelope's mouth. Successive happenings in this chain involve every last microparticle in every last antelope of that species alive at the time, and in every last blade of grass ingested over the next ten years by any of those antelopes. The chain catches each of these microparticles in just the right state-a state it is in, at the moment the causal chain catches up with it, precisely because of the state it had been in at the moment the chain started. The chain ends up sufficing for the occurrence of event $e$ in the eyelid of the newborn antelope. But indispensable for its causal sufficiency is the state each of the scattered microparticles was in, at the instant the chain started. That collection of microparticle states, as complex as it was, and as widely-scattered as the featured microparticles were, was as a whole a Necessary component of what Sufficed for $e$.

This scenario is closer to laughable than to believable. At the macro-level, it seems obvious that the causal chains which lead to the existence of the newborn antelope, eyelid and all, run through the lives of that newborn's forebears; just possibly through the lives, as well, of other antelopes who competed with the forebears for forage or mates; just possibly through the careers, as well, of all the blades of grass which the forebears ate. How astonishing if, on the level of microphysics, the causal chain that leads to $e$ runs far more widely: through the bodies of every member of the newborn's species, even completely unrelated ones, and through every microparticle in each of these bodies! It would be as if the microphysical chain were exquisitely sensitive to the membership boundaries of the newborn's species, and to the spatial boundaries of those members' bodies. It is far, far more believable that if the microparticle movement in the lucky antelope's mouth does launch a causal chain which results in $e$, that chain does not embroil every last microparticle featured in the cause collection. No state of that sprawling group of microparticles as a whole—no collection of states each involving one microparticle within that group - is causally indispensable for the production of $e$. The cause collection is not an NS 
condition of any component of the effect collection. So it does not cause the effect collection in piecemeal fashion.

Might nomological sufficiency be adequate to rescue physicalism? I have argued that there is no law of nature joining an antecedent which the cause collection as a whole satisfies with a consequent which the effect collection as a whole satisfies. There is a generalization which joins that antecedent to that consequent, but it is merely a theorem of the laws, rather than itself a law. But perhaps this concedes all the physicalist really needs. For it does now follow that the occurrence of the cause collection is nomologically sufficient for the occurrence, a certain interval later, of the effect collection. Doesn't this in effect concede that every causing rightly reported by the special sciences can be recaptured at the level of the microparticles?

Not recaptured as a causing, I maintain—not as one collection of microparticle happenings' bringing about another collection. Nomological sufficiency can after all obtain where causation plainly does not. Consider for example the sort of case in which a certain state of affairs- the presence of a certain disease in a person, say, or of a certain trace metal in a compound-manifests itself in two or more characteristic ways. Either manifestation can (in normal circumstances) get produced only if the other is produced as well. So the occurrence of either is nomologically sufficient for the other. But neither causes the other. Or consider any case in which (in standard circumstances, at least) a certain effect can be produced in only one way. Its occurrence is nomologically sufficient for the earlier event which caused it.

True causation tracks the contours of the laws of nature far more closely than nomological sufficiency does. It tracks them too closely, I have argued, for physicalism to be plausible. 
This paper argues against a thesis widely regarded as undeniable. Worse yet, physicalism is widely regarded as crucial for our understanding of how the various sciences relate to one another. It would be fair to ask whether an alternative understanding is in the offing, or merely a lack of understanding.

In crude terms - the best I can offer at this point-my alternative understanding runs this way. The objects of the world are composed of microparticles. Microparticles have their ways, but their ways do not exhaust the ways of the objects which they compose. Those objects are genuine wholes, not mere parcels or heaps of microparticles. Their trajectories and behaviors cannot be plotted by summing over the microparticles within them. For they can and do survive defections by individual component microparticles, and accretions of new ones, as mere parcels of microparticles cannot ${ }^{6}$ They have natures of their own. ${ }^{7}$ 


\section{Footnotes}

${ }^{1}$ The view most often is articulated as a claim that mental causation is shadowed, even rivaled, by causation at the level of microphysics. But arguments for that version of the view commonly entail that not just mental causation, but causation reported by any of the special sciences, is thus shadowed. See Sturgeon 1998, e.g. at p. 418 (“a physicalism that is both general and severe”), and cf. Yablo 1992a.

${ }^{2}$ D. M. Armstrong espouses a modest physicalism (see, for example, 1997, p. 153), though some of his formulations may sound like hegemonic physicalism instead (e.g. 1997, p. 6 or p. 253).

${ }^{3}$ Jaegwon Kim's arguments are among the most forceful there are for hegemonic physicalism, but Kim's own position is somewhat hedged: either the causings reported by the special sciences really are done by collections of microparticles instead, or there is no question of "instead"- the causes identified by the special sciences are just a matter of such collections of microparticles coming to satisfy very complex microphysical descriptions. See Kim 1989a and 1989b, or Kim forthcoming.

${ }^{4}$ Less stable mainly because of the problem of coinciding objects. If the medium-sized objects posited by the special sciences actually cause things, and figure in laws, they have as valid a claim to real existence, prima facie, as do the microparticles which compose them. But the parcel of microparticles which (it is said) composes any such object has very different modal properties from that object itselfmedium-sized objects are generally "mereologically incontinent", while parcels are "mereologically stable" (see, e.g., Zimmerman 1995). Modest physicalism may be less stable also because of the problem of harmonizing laws. If individual microparticles are bound only to obey the laws of microphysics, how can it be that parcels of microparticles cannot fail to obey laws belonging to different sciences altogether?

${ }^{5}$ The physicalist might concede—as, in section I, I suggest she should—-that some microparticle movements comprised in the so-called "cause collection" are "just along for the ride". Still, for the rest of the cause collection as a whole to qualify as an NS condition for e, every last one of the movements in the rest must indispensably have been involved in propagating the chain that terminated in $e$. 
${ }^{6}$ This may seem to raise what is called the problem of coinciding objects. If objects recognized by special sciences typically are "mereologically incontinent", while the parcel of microparticles which composes any such object is "mereologically stable", we seem to have two objects with different modal capacities occupying exactly the same spot. My solution is that there are microparticles, and mediumsized objects composed of microparticles, but no parcels of microparticles (Elder 1998).

${ }^{7}$ For comments on earlier drafts I am grateful to Anne Hiskes, Ruth Millikan, Austen Clark, and John Troyer. 


\section{References}

Armstrong, David 1997: A World of States of Affairs. Cambridge: Cambridge Univ. Press.

Bennett, Jonathan 1988: Events and Their Names. Indianapolis: Hackett.

Davidson, Donald 1967: “Causal Relations”. Journal of Philosophy, 64, pp. 691-703. 1989: “The Individuation of Events”. In Rescher 1969.

Elder, Crawford 1998: "Essential Properties and Coinciding Objects". Philosophy and Phenomenological Research, 58, pp. 317-31.

forthcoming: "Mental Causation versus Physical Causation: No Contest". Philosophy and Phenomenological Research.

Fodor, Jerry 1975: The Language of Thought. New York: Thomas Y. Crowell.

Kim, Jaegwon 1969: “Events and their Descriptions”. In Rescher 1969.

1980: "Events as Property Exemplifications". In Brand, Miles, and Walton, D., Action Theory. Dordrecht: D. Reidel.

1989a: "The Myth of Nonreductive Materialism". Proceedings and Addresses of the American Philosophical Association, 63, pp. 31-47.

1989b: "Mechanism, Purpose, and Explanatory Exclusion”. Philosophical Perspectives, 3, pp. $77-108$.

forthcoming: "Making Sense of Emergence". Forthcoming proceedings of the 1997 Oberlin Conference.

Millikan, Ruth 1993: "What is Behavior? A Philosophical Essay on Ethology and Individualism in Biology”. In her White Queen Psychology and Other Essays for Alice. Cambridge, Mass.: M.I.T. Press.

Neander, Karen 1991: “Misrepresenting and Malfunctioning”. Philosophical Studies, 79, pp. 109-41.

Rescher, Nicholas et al 1969: Essays in Honor of Carl Hempel. Dordrecht: D. Reidel. 
Sturgeon, Scott 1998: "Physicalism and Overdetermination". Mind, 107, pp. 411-32.

Unger, Peter 1980: "The Problem of the Many". In French, Peter A., Uehling, Theodore E., and Wettstein, Howard K., Midwest Studies in Philosophy, V: Studies in Epistemology. Minneapolis: Univ. of Minnesota Press.

van Inwagen, Peter 1990: Material Beings. Ithaca: Cornell Univ. Press.

Yablo, Stephen 1992a: "Mental Causation”. Philosophical Review, 101, pp. 245-80. 1992b: "Cause and Essence". Synthese, 93, pp. 403-49.

Zimmerman, Dean 1995: “Theories of Masses and Problems of Constitution”. Philosophical Review, 104, pp. 53-110. 